\title{
Modulation of ethylene- and heat-controlled hyponastic leaf movement in Arabidopsis thaliana by the plant defence hormones jasmonate and salicylate
}

\author{
Martijn van Zanten · Tita Ritsema • Joanna K. Polko • \\ Antonio Leon-Reyes • Laurentius A. C. J. Voesenek • \\ Frank F. Millenaar · Corné M. J. Pieterse • Anton J. M. Peeters
}

Received: 7 July 2011/Accepted: 25 September 2011/Published online: 19 October 2011

(C) The Author(s) 2011. This article is published with open access at Springerlink.com

\begin{abstract}
Upward leaf movement (hyponastic growth) is adopted by several plant species including Arabidopsis thaliana, as a mechanism to escape adverse growth conditions. Among the signals that trigger hyponastic growth are, the gaseous hormone ethylene, low light intensities, and supra-optimal temperatures (heat). Recent studies
\end{abstract}

M. van Zanten $(\square) \cdot$ J. K. Polko · L. A. C. J. Voesenek ·

F. F. Millenaar - A. J. M. Peeters

Plant Ecophysiology, Institute of Environmental Biology,

Utrecht University, Padualaan 8, 3584 CH Utrecht,

The Netherlands

e-mail: m.vanzanten@uu.nl

M. van Zanten

Department of Plant Breeding and Genetics,

Max Planck Institute for Plant Breeding Research,

Carl-von-Linné-Weg 10, 50829 Cologne, Germany

T. Ritsema · A. Leon-Reyes - C. M. J. Pieterse

Plant-Microbe Interactions, Institute of Environmental Biology,

Utrecht University, Padualaan 8, 3584 CH Utrecht,

The Netherlands

Present Address:

T. Ritsema

AMT, Meibergdreef 61, 1105 BA Amsterdam, The Netherlands

Present Address:

A. Leon-Reyes

Universidad San Francisco de Quito, Diego de Robles Y Vía

Interoceánica (Cumbaya), 17-1200-841 Quito, Ecuador

L. A. C. J. Voesenek - C. M. J. Pieterse

Centre for BioSystems Genomics, P.O. Box 98, 6700 AB

Wageningen, The Netherlands

Present Address:

F. F. Millenaar

Monsanto, Leeuwenhoekweg 52, 2660 BB Bergschenhoek,

The Netherlands indicated that the defence-related phytohormones jasmonic acid (JA) and salicylic acid (SA) synthesized by the plant upon biotic infestation repress low light-induced hyponastic growth. The hyponastic growth response induced by high temperature (heat) treatment and upon application of the gaseous hormone ethylene is highly similar to the response induced by low light. To test if these environmental signals induce hyponastic growth via parallel pathways or converge downstream, we studied here the roles of Methyl-JA (MeJA) and SA on ethylene- and heatinduced hyponastic growth. For this, we used a time-lapse camera setup. Our study includes pharmacological application of MeJA and SA and biological infestation using the JA-inducing caterpillar Pieris rapae as well as mutants lacking JA or SA signalling components. The data demonstrate that MeJA is a positive, and SA, a negative regulator of ethylene-induced hyponastic growth and that both hormones repress the response to heat. Taking previous studies into account, we conclude that SA is the first among many tested components which is repressing hyponastic growth under all tested inductive environmental stimuli. However, since MeJA is a positive regulator of ethyleneinduced hyponastic growth and is inhibiting low light- and heat-induced leaf movement, we conclude that defence hormones control hyponastic growth by affecting stimulusspecific signalling pathways.

Keywords Arabidopsis - Ethylene $\cdot$ Heat $\cdot$ Hyponastic growth $\cdot$ Jasmonate $\cdot$ Leaf movement $\cdot$ Salicylate
Abbreviations
JA Jasmonic acid
SA Salicylic acid
MeJA Methyl-jasmonic acid
ABA Phytohormone abscisic acid 
nprl-1 Non-expresser of PR genes 1 mutant

coil-1 Coronatine insensitive1-1 mutant

\section{Introduction}

Upward leaf movement (hyponastic growth), driven by differential cell elongation in the petiole is a mechanism exploited by several plant species to outgrow growth hampering environmental conditions (Voesenek et al. 2006; van Zanten et al. 2010a; Polko et al. 2011). Recent studies have indicated that in Arabidopsis thaliana hyponastic growth is induced upon exposure to ethylene (Millenaar et al. 2005, 2009; van Zanten et al. 2010a, b), low light conditions (Millenaar et al. 2005, 2009; Mullen et al. 2006; Ritsema et al. 2010) and high temperatures (heat) (Koini et al. 2009; van Zanten et al. 2009a; Vasseur et al. 2011). All these signals induce hyponastic growth within $1 \mathrm{~h}$ and the kinetics of the response to these signals are remarkably similar. Therefore, it was proposed that these three parallel signalling pathways may merge downstream and operate a shared set of functional hyponastic growth-associated genetic components (van Zanten et al. 2010a). Yet, among all hormonal interactions and genetic components that so far have been described to control hyponastic leaf movements in Arabidopsis, only gibberellin was shown to be a synonymous (positive) controller of the response to all treatments (PeñaCastro et al. 2011). For example, mutant and pharmacological analyses have shown that low light-induced hyponastic growth is independent of ethylene action, whereas heatinduced hyponastic growth is inhibited by this gaseous hormone (van Zanten et al. 2009a). Auxin and polar auxin transport do not seem to play a direct role in ethyleneinduced hyponastic growth (van Zanten et al. 2009b), but are required for the response induced by low light and elevated temperatures (Millenaar et al. 2005; Koini et al. 2009; van Zanten et al. 2009a). Abscisic acid (ABA) antagonizes ethylene-induced hyponastic growth (Benschop et al. 2007) and is a positive regulator of heat-induced hyponastic growth (van Zanten et al. 2009a).

Recently, we demonstrated that the phytohormones, methyl jasmonic acid (MeJA) and salicylic acid (SA), that direct induced defence against various biotic attackers in plants (Glazebrook 2005) are negative regulators of hyponastic growth induced by low light intensities (Ritsema et al. 2010). This interaction between light signalling and defence hormones was predicted using kinase substrate peptide-containing PepChip arrays.

Since the kinetics and amplitude of the hyponastic growth response induced by ethylene, low light and heat are highly similar and the signal cascades may merge downstream, we tested if MeJA and SA repress hyponastic growth induced by ethylene and heat in a similar way as in low light-induced hyponastic growth (Ritsema et al. 2010). Our results, however, demonstrate that MeJA is a positive, and SA, a negative regulator of ethylene driven hyponastic growth in a dosedependent manner. Both hormones repress the response to heat treatment. Our data suggest complex cross talk between the biotic and abiotic environment at multiple levels in the signal transduction towards hyponastic growth. Because MeJA has a positive effect on ethylene-induced hyponastic growth and is inhibiting low light- and heat-induced hyponastic growth, we conclude that defence hormones at least partly affect different signalling components/pathways towards induction of hyponastic growth and that the specific role the defence-related hormones play in the control of leaf movement thus depends on the hyponastic growth-inducing stimulus. Nevertheless, SA is the first identified component which represses hyponastic growth under all tested inducing environmental stimuli (i.e. ethylene, low light and heat).

\section{Materials and methods}

Plant material and growth conditions

Arabidopsis thaliana L. Col-0 (N1092) and Col-5 (N1644) seeds were obtained from the Nottingham Arabidopsis Stock Centre. Nprl-1 is described in Cao et al. (1994), coil-1 is from Feys et al. (1994) and is in the Col-gl with gl1 marker genetic background (alias Col-5). Before potting, the seeds were stratified in the dark at $4^{\circ} \mathrm{C}$ for 4 days to synchronize germination. Because coil-1 is male-sterile, this line was propagated in a heterozygous state. Mutant coil-1 plants were grown on $1 / 2$ Murashige and Skoog medium containing $1 \%$ sucrose and $0.01 \mathrm{mM} \mathrm{MeJA}$ before use, and JA resistant plants were selected and transplanted to the soil mixture based on their root phenotype.

Plants were grown on a fertilized mixture of pot-soil and perlite $(1: 2 \mathrm{v} / \mathrm{v})$ as described previously by Millenaar et al. (2005) in $20^{\circ} \mathrm{C}, 70 \%$ (v/v) relative humidity, $200 \mu \mathrm{mol}$ $\mathrm{m}^{-2} \mathrm{~s}^{-1}$ photosynthetic active radiation (PAR), $9 \mathrm{~h}$ shortday photoperiod. Plants were automatically saturated with water each day, at the start of the photoperiod.

\section{Treatments}

Plants in developmental stage 1.10-1.12, according to Boyes et al. (2001) were used for all the experiments. Plants subjected to JA and SA treatment were withheld from water 3 days prior to the experiment and were transferred to the experimental setup 1 day before the experiment started to allow acclimatization. Treatments started $(t=0) 1.5 \mathrm{~h}$ after the start of the photoperiod to minimize diurnal and/or circadian effects. 
Ethylene was applied in continuous flow-through. In the camera setup, plants were positioned in glass cuvettes containing one plant each, as described in Millenaar et al. (2005, 2009) and Benschop et al. (2007). For the doseresponse measurements, plants were placed in a Microclima 1750 growth cabinet (Snijders Scientific, Tilburg, The Netherlands).

Ethylene (Hoek Loos, Amsterdam, The Netherlands) and air $[70 \%(\mathrm{v} / \mathrm{v})$ relative humidity] were mixed using flow meters to generate a concentration of $5 \mu 1^{-1}$ (camera setup) and $1.5 \mu \mathrm{l}^{-1}$ (dose-response experiments) which are saturating for the hyponastic response (Polko et al. 2011). The ethylene concentration was checked regularly on a gas chromatograph (GC955, Synspec, Groningen, The Netherlands) and remained constant for the duration of the experiment. Control plants were flushed with air $[70 \%$ (v/v) relative humidity] at the same flow rate. Heat treatment consisted of a sudden temperature increase, from $20^{\circ} \mathrm{C}$ (control) to $38^{\circ} \mathrm{C}$. This was accomplished by adjusting the programme of the used growth cabinet.

MeJA (Serva, Heidelberg, Germany) was dissolved in ethanol and subsequently diluted in water (milliQ) to the required concentration. SA (Duchefa, Haarlem, The Netherlands) was dissolved in water and ethanol was added to the SA solution to allow direct comparison with MeJA experiments. Mock solutions only contained ethanol. The standard mock solution, $100 \mu \mathrm{M}$ MeJA and $1 \mathrm{mM}$ all contained $1 \%$ ethanol. All solutions were added to the soil until saturation 1 hour prior to the start of the experiment (0.5 h after start of the photoperiod).

Pieris rapae larvae were raised essentially as described in De Vos et al. (2005). Three first-instar larvae were applied to each plant, $20 \mathrm{~h}$ before the start of the treatment with a small paintbrush. The control solution for the $P$. rapae experiments was MilliQ purified water.

\section{Computerized image analysis of angle kinetics} and calculations

Plants used for dose-response experiments were manually photographed from the side. Angles were measured using the freeware algorithm; ImageJ (http://rsb.info.nih.gov/ij). For all replicate plants, two representative petioles were measured and the values were averaged before subjection to further analysis. At least 12 plants (24 petioles) were used for each tested hormone concentration.

Angle kinetics experiments were conducted using an automated time-lapse camera setup as described in Millenaar et al. (2005). Per treatment and data point, 12-28 petioles of 6-18 individually grown plants have been measured, derived from at least two independently grown batches. Plants were placed individually in glass cuvettes with the petiole of study perpendicular to the axis of the camera. Petioles were marked at the petiole/lamina junction with orange paint (Decofin Universal, Apeldoorn, The Netherlands). These preparations did not influence the response of the petiole (data not shown). The light regime in the cuvettes was kept the same as during the growth period. Pictures of two petioles per plant were taken every $10 \mathrm{~min}$. To enable continuous photography, no dark period was included in the $24 \mathrm{~h}$ experimental period. Angles were measured between the orange painted point at the petiole/ lamina junction and a fixed basal point of the petiole and compared to the horizontal, by using KS400 (Version 3.0) software package (Carl Zeiss Vision, Hallbergmoos, Germany) and a customized macro. Pair wise subtraction was performed to take into account, the circadian and/or diurnal variations in petiole angle during the course of the experiments. For this, we calculated the difference between the angles of treated and control plants for each time point (Benschop et al. 2007). Calculation of the new standard error for the differential response was performed by taking the squared root from the summation of the two squared standard errors.

\section{Results}

MeJA and SA have opposing, dose-dependent effects on ethylene-induced hyponastic growth and repress heat-induced hyponastic growth

Our previous study indicated that MeJA and SA repress hyponastic growth induced by low light intensity and that the combination of these treatments (MeJA/SA) led to the abolishment of this leaf movement response (Ritsema et al. 2010). To allow the study of the effects of MeJA and SA on hyponastic growth induced by ethylene, we first applied different concentrations of MeJA and SA to A. thaliana accession Columbia-0 (Col-0) and monitored the hyponastic growth response after $9 \mathrm{~h}$ of ethylene treatment. In the absence of MeJA or SA pre-treatment, ethylene induced a leaf movement response of $\sim 12^{\circ}$ (Fig. 1). MeJA enhanced the response to ethylene in a dose-response manner, reaching a maximum angle difference of $\sim 30^{\circ}$ in the range $\sim 100-175 \mathrm{mM}$ JA. Higher concentrations $(>175 \mathrm{mM})$ led to a repression of the stimulating effect (Fig. 1a). In contrast, SA pre-treatment led to inhibition of ethylene-induced hyponastic growth at all concentrations and the response saturated at $1 \mathrm{mM} \mathrm{SA}$ (Fig. 1b).

To avoid detrimental effects of high MeJA concentrations, we used $100 \mathrm{mM}$ for the subsequent experiments in which the kinetics of the leaf movement was measured using a time-lapse camera setup. These experiments confirmed that pre-treatment with MeJA led to a strongly enhanced ethylene-induced hyponastic growth response, 

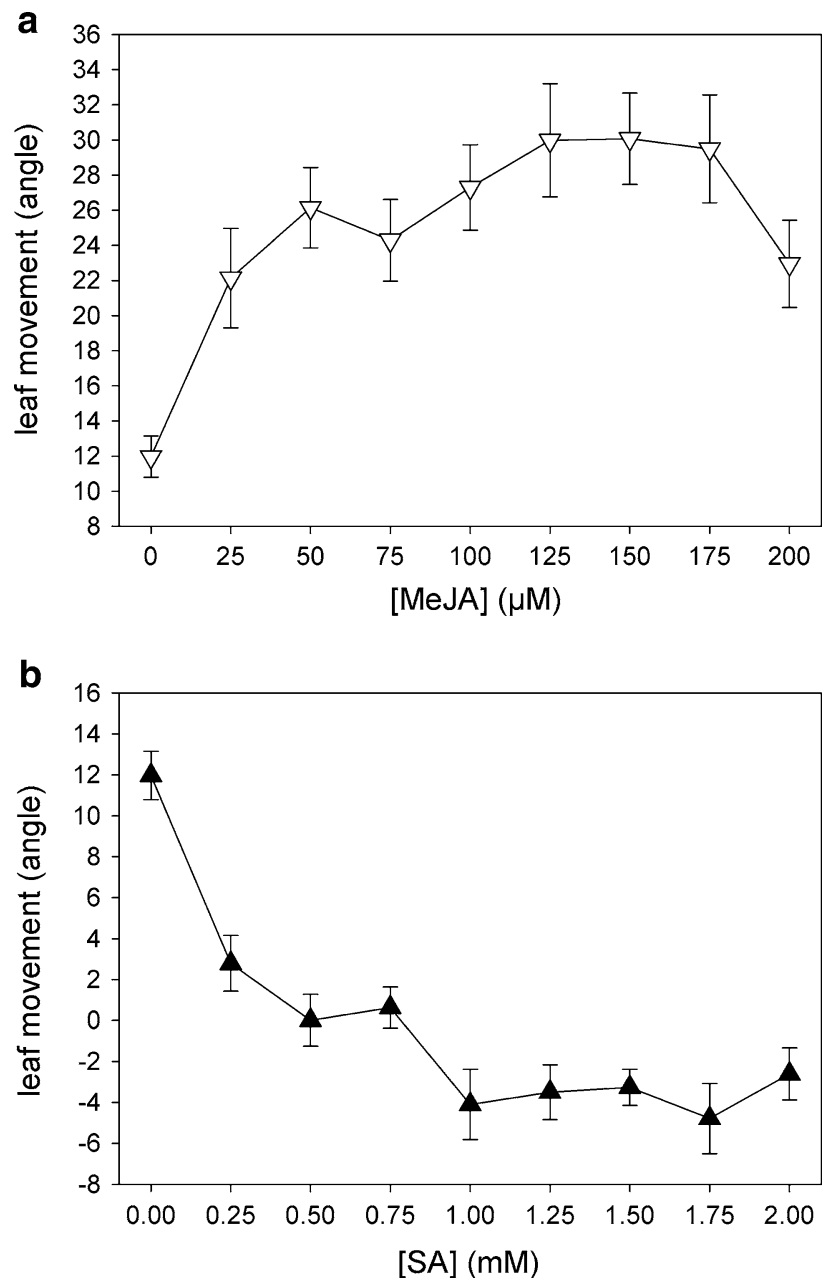

Fig. 1 MeJA and SA dosage effect on ethylene-induced hyponastic growth. Depicted angles represent the absolute difference in leaf angle before $(0 \mathrm{~h})$ and after $(9 \mathrm{~h})$ treatment with ethylene enriched $\left(1.5 \mu 11^{-1}\right)$ air, in the presence of different MeJA (a) or SA (b) concentrations. Note that the standard hyponastic growth response to ethylene treatment can be seen at concentration 0. Error bars represent $\mathrm{SE}, n>12$

starting approximately $4 \mathrm{~h}$ after ethylene treatment and lasting throughout the experimental period (Fig. 2a). Similar results were obtained when plants were infested with caterpillars of the JA-inducing herbivore $P$. rapae (Fig. 2b). The specificity of the hormonal action was tested with the aid of specific mutants. MeJA was unable to affect ethylene-induced hyponastic growth in the JA-insensitive coronatine insensitive1-1 (coil-1) receptor mutant (Fig. 2c, in the Col-gl/Col-5 genetic background). This shows that intact JA signalling is required for MeJA effects on hyponastic growth, but that the sensitivity for ethylene is not altered in this mutant. In contrast, the SAinsensitive mutant non-expresser of PR genes 1 (nprl-1) showed an enhanced ethylene-induced hyponastic growth response upon MeJA pre-treatment, similar as in wild type (Fig. 2d).
An opposite specificity was found for SA. Pre-treatment with $1 \mathrm{mM}$ SA suppressed ethylene-induced hyponastic growth in Col-0 (Fig. 2e). Similar to MeJA, the effects could be noticed approximately $4 \mathrm{~h}$ after the start of ethylene treatment and lasted throughout the experimental period (Fig. 2b). The repressing effect of SA as observed in wild type was also apparent in the coil-1 mutant background (Fig. 1f), whereas ethylene-induced hyponastic growth in the nprl-1 mutant was not inhibited by SA (Fig. 2g). When a combination treatment of SA and MeJA (SA/MeJA) was applied, this resulted in a repressed ethylene-induced hyponastic growth response reminiscent of the reaction to SA in wild-type plants (Fig. 2h).

In contrast to the observed effect of MeJA on ethyleneinduced hyponastic growth, pre-treatment with MeJA suppressed the hyponastic growth response to heat treatment $\left(38^{\circ} \mathrm{C}\right)$ (Fig. 3a). Similarly, SA also repressed the response to heat in Col-0 (Fig. 3b). Simultaneous application of SA and MeJA resulted in the abolishment of the response to heat treatment (Fig. 3c).

Together, these data demonstrate that phytohormones produced upon pathogen or insect attack influence leaf movement responses in a complex manner. Our data suggest that SA and MeJA act additive in inhibiting heat-induced hyponastic growth and that SA-mediated repression is dominant over the enhancing effects of MeJA on ethyleneinduced hyponasty.

\section{Discussion}

In this work we demonstrate that the defence-related phytohormones MeJA and SA control upward leaf movement induced by ethylene and high temperature (heat) in $A$. thaliana Col-0. Studies on the interaction between the expression of defence and the responses to the abioic environment mainly focused on neighbour competition and the associated changes in spectral quality perceived by the plant. This interrelation is therefore now relatively well understood (reviewed in Ballaré 2011; Kazan and Manners 2011). For example, MeJA and SA have been associated with the so-called shade avoidance syndrome (Kurashige and Agrawal 2005; McGuire and Agrawal 2005; FaigonSoverna et al. 2006; Izaguirre et al. 2006; Moreno et al. 2009), which is a suite of traits induced in dense canopies as a mechanism to outgrow the neighbouring competitors. Hyponastic growth is among the most striking component traits of the shade avoidance syndrome in rosette species and both MeJA and SA repress hyponastic growth induced by low light intensity (without changing the spectral composition of the light) in A. thaliana Col-0, whereas the combined treatment even abolished the response (Ritsema et al. 2010). In addition, constitutive shade-avoiding 

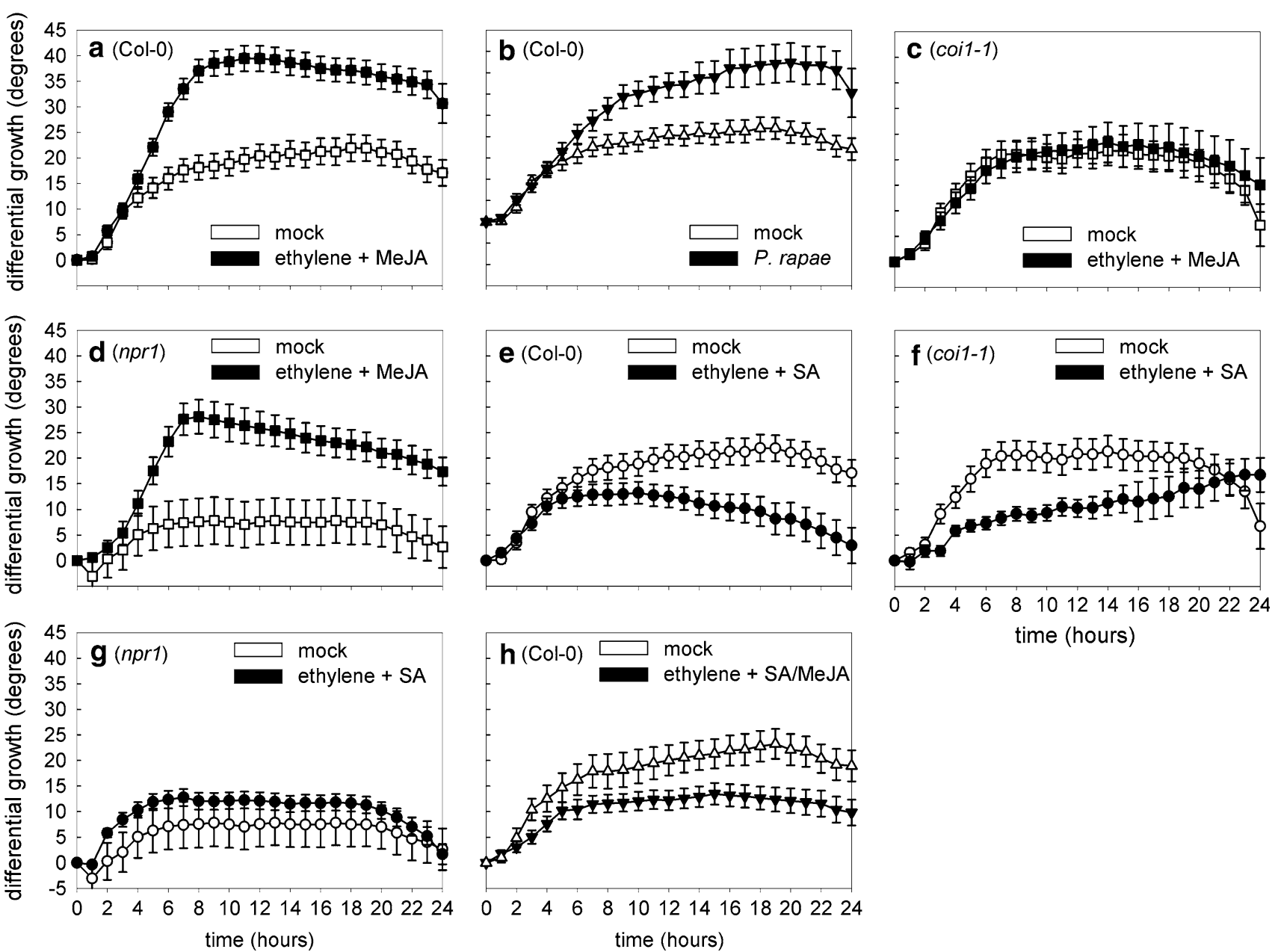

symbols) on differential growth of Arabidopsis petioles (angles) in ethylene enriched air $\left(5 \mu 11^{-1}\right)$. a, b, e, h Col-0 wild type. c, f coil-1. d, g npr1-1. Error bars represent SE, $n>12$. Data are pair wise subtracted, which is the difference between the angles of treated and control plants for each time point (Benschop et al. 2007)

mutant plants are more susceptible to herbivore attack than wild-type plants (McGuire and Agrawal 2005; FaigonSoverna et al. 2006) and plants subject to light competition or spectral shade allow a better survival and growth of caterpillars (Kurashige and Agrawal 2005; Izaguirre et al. 2006). Pseudomonas-induced SA signalling was found to depend on the light and day length after inoculation, and is specifically dependent on phytochrome-mediated signalling pathways (Genoud et al. 2002; Griebel and Zeier 2008).

In this work we showed that besides interactions with low light signalling, MeJA and SA also modulate ethyleneinduced hyponastic growth which is associated with submergence avoidance (Voesenek et al. 2006). Under complete flooding, this phytohormone becomes entrapped in plants and is the main trigger for hyponastic growth in several semi-aquatic species to restore gas-exchange with the environment by reaching above the water table. This

trait is conserved in Arabidopsis (Millenaar et al. 2005, 2009). Heat-induced hyponasty in Arabidopsis is assumed to be an adaptation to reduce the heat flux of direct sunlight and enhances evaporative cooling (van Zanten et al. 2009a, 2010a; Vasseur et al. 2011). Taken together, our results suggest that cross talk between defence signalling and abiotic signals is not limited to neighbour competition and low light signalling only, but likely can be broadened to various relevant natural situations in which multiple stresses are simultaneously imposed on the plant.

Different pathogens and herbivore insects induce different blends of defence hormones in the plant. Moreover, these hormones influence each other's action (Pieterse et al. 2009). For example, SA is induced upon the attack of biotrophic pathogens, and MeJA is synthesized upon insect herbivory (Glazebrook 2005; De Vos et al. 2005). The type of the infesting pathogen and parameters in the abiotic environment therefore highly determine the nature of the 


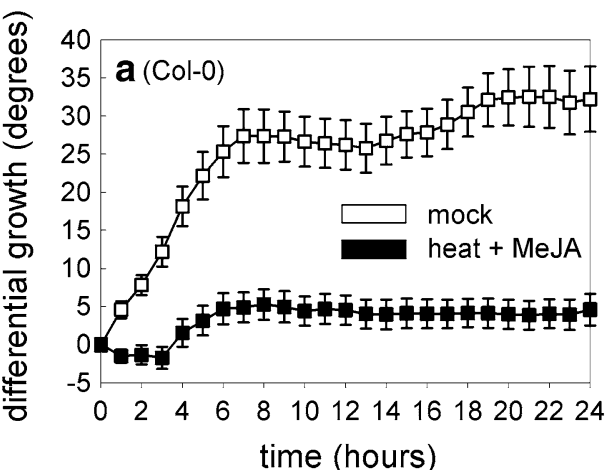

Fig. 3 MeJA and SA effects on heat-induced hyponastic growth. a Effect of $(1 \mathrm{~h})$ pre-treatment with $100 \mu \mathrm{M}$ MeJA (closed squares). b $1 \mathrm{mM} \mathrm{SA}$ (closed circles) on Arabidopsis Col-0 petiole angles of

leaf angle phenotype. As mentioned before, the hyponastic growth response to the different environmental signals (low light, heat, ethylene) is tightly controlled by a blend of several phytohormones (reviewed in van Zanten et al. 2010a), which includes gibberellins and auxins, hormones that now also have been associated to defence signalling (reviewed in Ballaré 2011; Kazan and Manners 2011). Given these complex multi-level interactions, it is very delicate to speculate on the functional, ecological, and evolutionary implications, and the mechanism(s) of the interaction between defence hormones and hyponastic growth. However, it is apparent that MeJA and SA have important functions in inducing, guarding, and fine-tuning responses to the biotic and abiotic environment. Obtaining full understanding of the functional mechanistic details and ecological implications would require mathematical modelling. Quantification of the effects of JA and SA on leaf angles under different environmental stresses as presented in this study and in Ritsema et al. (2010) would be useful for such an approach.

Based on the data presented in this paper, in Ritsema et al. (2010) and the parsimonious model presented in our recent review (van Zanten et al. 2010a), we propose a model how hyponastic growth induced by different environmental signals is modulated by JA and SA (Fig. 4).

MeJA and SA enhance and repress the hyponastic response induced by ethylene in a COI1 and NPR1 dependent manner, respectively (Fig. 2). The enhancing effect of MeJA on ethylene-induced hyponastic growth is in sharp contrast to what we observed previously for low light (Ritsema et al. 2010) and heat (Fig. 3). This indicates that hyponastic growth induced by different environmental signals is, at least partly, regulated via distinct pathways by these defence-related hormones (Fig. 4). SA appears to act dominantly over MeJA during ethylene-induced hyponastic growth, as a repressed hyponastic growth response was observed in a combined treatment SA/MeJA, reminiscent

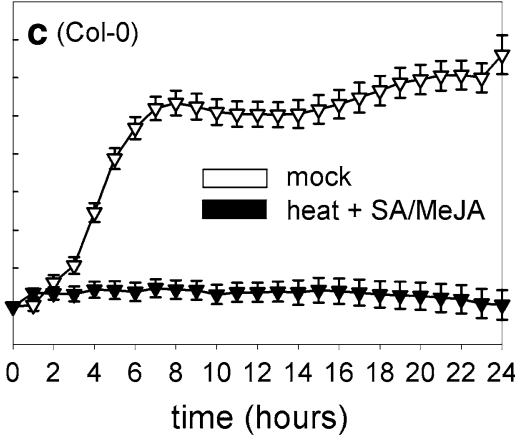

plants subjected to heat treatment (shift from 20 to $38^{\circ} \mathrm{C}$ ). Error bars represent SE, $n>12$. See Fig. 2 for more details

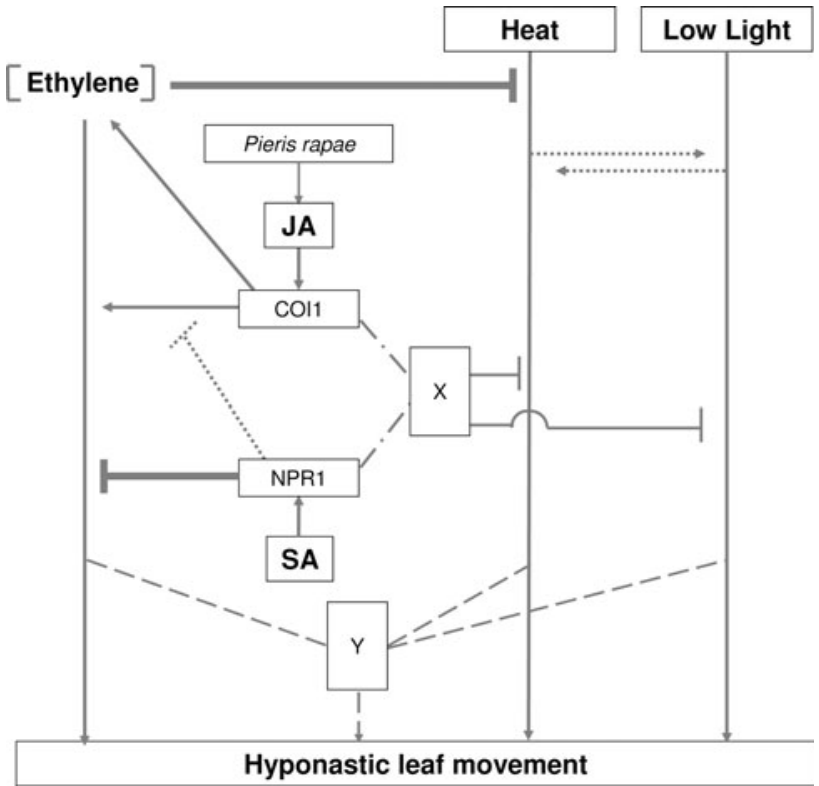

Fig. 4 Proposed signalling network of JA and SA modulation of ethylene, heat and low light-induced hyponastic petiole growth. See text for details. Model based on the parsimony model presented in van Zanten et al. (2010a). Note that order of the signals along the arrows does not necessarily reflect the order of interactions occurring. Bold lines indicate dominant effects. Dotted lines between heat and low light signalling indicate that we cannot distinguish if low light sensitizes the plant for heat or vice versa. The box indicated by X represents a putative integrative factor downstream of JA and SA signalling that controls heat- and low light-induced hyponastic growth in an additive manner. The dotted line between NPR1 and COI1 action indicates possible inhibition of JA signalling via NPR1 dependent SA signalling. The dashed lines and the box indicated by $\mathrm{Y}$ at the bottom indicate the likely convergence of downstream signalling pathways in which the same functional genetic components are utilized by the different signals to control hyponastic growth (see also van Zanten et al. 2010a). However, we cannot rule out the existence of independent inducing-stimulus dependent parallel signalling cascades towards hyponastic growth

to single application of SA. SA is known to inhibit JA signalling in an NPR1-dependent manner and this likely contributes to the observed effect (Spoel et al. 2003). 
It has been shown before that ethylene modulates the effects of SA and JA (Glazebrook et al. 2003; Glazebrook 2005; Leon-Reyes et al. 2009). SA-dependent defence is generally stimulated by ethylene. The situation with MeJA is more complex, the wound response is repressed, whereas the so-called pathogen response is enhanced by ethylene (Thatcher et al. 2005; Leon-Reyes et al. 2009). JA, together with an ethylene burst, is predominantly induced upon necrotrophic pathogen infestation. It should be noted in this context that heat represses ethylene production and that ethylene is a dominant negative regulator of heat-induced hyponastic growth (van Zanten et al. 2009a). Since no interaction between low light and ethylene was found (Millenaar et al. 2009), ethylene accumulation cannot interfere with the JA- and MeJA/SA-mediated effects to shade. This indicates that JA and SA may affect the same unknown downstream regulator that controls the hyponastic growth to low light and heat, but not ethylene (indicated by Box X in Fig. 4), and that these signals do so in an additive manner since the combined effect of MeJA/ $\mathrm{SA}$ is the abolishment of the hyponastic response. This is in the assumption that NPR1 and COI1 are required for, respectively, SA- and JA-mediated inhibition of heatinduced hyponastic growth, which is highly likely, given their requirement for SA and JA effects on ethyleneinduced (Fig. 2) and low light-induced (Ritsema et al. 2010) hyponastic growth. The results here presented on SA are in line with the observations that protection against heat stress (oxidative damage to membranes) involves SA (Larkindale and Knight 2002).

Taken together, the effects of MeJA, SA, and SA/MeJA application on heat- and low light-induced hyponastic growth are highly comparable and differ from the effects of MeJA on ethylene-induced hyponastic growth (Fig. 4). This is in line with the notion that ethylene and low light signalling towards differential petiole growth are due to largely independent signal transduction cascades (Millenaar et al. 2005, 2009) and is in accordance with the observation that heat- and low light-induced hyponastic are synonymously controlled by several (other) phytohormones (i.e., auxin and ABA; reviewed in van Zanten et al. 2010a) and act additively (van Zanten et al. 2009a).

SA single treatment represses hyponastic growth induced by ethylene, low light, and heat and thus seems to be a general repressor of hyponastic growth irrespective of the inducing signal. This conclusion is significant because, based on the high similarity in the kinetics of the hyponastic growth response, it was postulated that the signal transduction routes induced by different stimuli integrate downstream towards induction of hyponastic growth and probably target the same functional mechanism(s) (van Zanten et al. 2010a). However, to date, none of the phytohormones or genetic components described to control hyponastic growth in Arabidopsis has been shown to do so in a synonymous negative manner (Mullen et al. 2006; Benschop et al. 2007; Millenaar et al. 2009; van Zanten et al. 2010a, b). Testing if down regulation of the SA level is an integral part of the induction of hyponastic growth to all these treatments would require direct measurements of SA levels in response to these treatments. However, as hyponastic growth is typically induced already within an hour after start of the treatment (Millenaar et al. 2009) and given that small amounts of SA already strongly affect the hyponastic growth response (Fig. 1), we conclude that the leaf movement response is likely induced much faster than the time required for complete metabolic turnover of the SA compound.

We demonstrated that the enhancing effect of MeJA on ethylene-induced hyponastic growth was absent specifically in the JA-insensitive coil-1 mutant and that the repressing SA effect was absent specifically in the SAinsensitive nprl-1 mutant. This shows that both phytohormones require intact but different signalling routes, which add to the suggestion that at least upstream hormone-specific pathways to exert effects on hyponastic growth differ. Also, taking into account that the combined SA/MeJA application had different effects than the single application, we conclude that JA and SA likely affect a common downstream target(s) in an additive manner in the control of hyponastic growth (Box Y in Fig. 4).

It can be seen in Fig. $2 \mathrm{~d}$, g that the nprl mutant has reduced ethylene-induced hyponastic growth. This suggests that the ethylene-induced hyponastic growth requires NPR1, which is normally identified as an essential component of SA signalling. Although the exact interpretation for the apparent reduced hyponasty when ethylene is added to nprl mutant plants is unclear, direct interactions between ethylene and NPR1 have been observed before. For example, it was shown that ethylene can bypass the need for NPR1 in the cross talk between SA and JA (LeonReyes et al. 2009), indicating that NPR1 and ethylene interact directly without the need for SA. In the same paper, it was shown that this ethylene effect is dependent on the ethylene receptor protein ETHYLENE INSENSITIVE 2 (EIN2). Furthermore, it was speculated that this effect of NPR1 is served by cytosolic NPR1, whereas its role in SA-mediated transcription requires the protein to travel to the nucleus. Also indicative for an SA-independent function of NPR1, and a possible interference with ethylene signalling is a role for NPR1 in JA- and ethylenedependent resistance against the pathogenic fungus Verticillium longisporum (Johansson et al. 2006).

Acknowledgments This work was supported by the Dutch Organization for Scientific Research (NWO) TI Pharma [Grant number T3-103 to TR] and VICI (NWO) [Grant Number 865.04.002 to CMJP]. We thank Dr. Ronald Pierik for helpful comments on the manuscript. 
Open Access This article is distributed under the terms of the Creative Commons Attribution Noncommercial License which permits any noncommercial use, distribution, and reproduction in any medium, provided the original author(s) and source are credited.

\section{References}

Ballaré CL (2011) Jasmonate-induced defenses: a tale of intelligence, collaborators and rascals. Trends Plant Sci 16:249-257

Benschop JJ, Millenaar FF, Smeets ME, van Zanten M, Voesenek LACJ, Peeters AJM (2007) Abscisic acid antagonizes ethyleneinduced hyponastic growth in Arabidopsis. Plant Physiol 143:1013-1023

Boyes DC, Zayed AM, Ascenzi R (2001) Growth stage-based phenotypic analysis of Arabidopsis: a model for high throughput functional genomics in plants. Plant Cell 13:1499-1510

Cao H, Bowling SA, Gordon AS, Dong XN (1994) Characterization of an Arabidopsis mutant that is nonresponsive to inducers of systemic acquired resistance. Plant Cell 6:1583-1592

De Vos M, Van Oosten VR, Van Poecke RMP, Van Pelt JA, Pozo MJ, Mueller MJ, Buchala AJ, Metraux JP, Van Loon LC, Dicke M, Pieterse CMJ (2005) Signal signature and transcriptome changes of Arabidopsis during pathogen and insect attack. Mol Plant Microbe Interact 18:923-937

Faigon-Soverna A, Harmon FG, Storani L, Karayekov E, Staneloni RJ, Gassmann W, Mas P, Casal JJ, Kay SA, Yanovsky MJ (2006) A constitutive shade-avoidance mutant implicates TIRNBS-LRR proteins in Arabidopsis photomorphogenic development. Plant Cell 18:2919-2928

Feys B, Benedetti CE, Penfold CN, Turner JG (1994) Arabidopsis mutants selected for resistance to the phytotoxin coronatine are male sterile, insensitive to methyl jasmonate, and resistant to a bacterial pathogen. Plant Cell 6:751-759

Genoud T, Buchala AJ, Chua NH, Metraux JP (2002) Phytochrome signalling modulates the SA-perceptive pathway in Arabidopsis. Plant J 31:87-95

Glazebrook J (2005) Contrasting mechanisms of defense against biotrophic and necrotrophic pathogens. Ann Rev Phytopathol 43:205-227

Glazebrook J, Chen W, Estes B, Chang HS, Nawrath C, Mtraux JP, Zhu T, Katagiri F (2003) Topology of the network integrating salicylate and jasmonate signal transduction derived from global expression phenotyping. Plant J 34:217-228

Griebel T, Zeier J (2008) Light regulation and daytime dependency of inducible plant defences in Arabidopsis: phytochrome signalling controls systemic acquired resistance rather than local defence. Plant Physiol 147:780-790

Izaguirre MM, Mazza CA, Biondini M, Baldwin IT, Ballaré CL (2006) Remote sensing of future competitors: impacts on plant defenses. Proc Natl Acad Sci USA 103:7170-7174

Johansson A, Staal J, Dixelius C (2006) Early responses in the Arabidopsis-Verticillium longisporum pathosystem are dependent on NDR1, JA- and ET-associated signals via cytosolic NPR1 and RFO1. Mol Plant Microbe Interact 19:958-969

Kazan K, Manners JM (2011) The interplay between light and jasmonate signalling during defence and development. J Exp Bot 62:4087-4100

Koini MA, Alvey L, Allen T, Tilley CA, Harberd NP, Whitelam GC, Franklin KA (2009) High temperature-mediated adaptations in plant architecture require the bHLH transcription factor PIF4. Curr Biol 19:408-413

Kurashige NS, Agrawal AA (2005) Phenotypic plasticity to light competition and herbivory in Chenopodium album (Chenopodiaceae). Am J Bot 92:21-26
Larkindale J, Knight MR (2002) Protection against heat stressinduced oxidative damage in Arabidopsis involves calcium, abscisic acid, ethylene, and salicylic acid. Plant Physiol 128:682-695

Leon-Reyes A, Spoel SH, De Lange ES, Abe H, Kobayashi M, Tsuda $\mathrm{S}$, Millenaar FF, Welschen RAM, Ritsema T, Pieterse CMJ (2009) Ethylene modulates the role of NONEXPRESSOR OF PATHOGENESIS-RELATED GENES1 in cross talk between salicylate and jasmonate signaling. Plant Physiol 149:1797-1809

McGuire R, Agrawal AA (2005) Trade-offs between the shadeavoidance response and plant resistance to herbivores? Tests with mutant Cucumis sativus. Funct Ecol 19:1025-1031

Millenaar FF, Cox MC, Van Berkel YE, Welschen RA, Pierik R, Voesenek LACJ, Peeters AJM (2005) Ethylene-induced differential growth of petioles in Arabidopsis. Analyzing natural variation, response kinetics, and regulation. Plant Physiol 137:998-1008

Millenaar FF, van Zanten M, Cox MCH, Pierik R, Voesenek LACJ, Peeters AJM (2009) Differential petiole growth in Arabidopsis thaliana: photocontrol and hormonal regulation. New Phytol 184:141-152

Moreno JE, Tao Y, Chory J, Ballaré CL (2009) Ecological modulation of plant defense via phytochrome control of jasmonate sensitivity. Proc Natl Acad Sci USA 106:4935-4940

Mullen JL, Weinig C, Hangarter RP (2006) Shade avoidance and the regulation of leaf inclination in Arabidopsis. Plant Cell Environ 29:1099-1106

Peña-Castro J, van Zanten M, Lee SC, Patel M, Voesenek LACJ, Fukao T, Bailey-Serres J (2011) Expression of rice SUB1A and $S U B 1 C$ transcription factors in Arabidopsis uncovers flowering inhibition as a submergence-tolerance mechanism. Plant $\mathrm{J}$ 67:434-446

Pieterse CM, Leon-Reyes A, Van der Ent S, Van Wees SC (2009) Networking by small-molecule hormones in plant immunity. Nat Chem Biol 5:308-316

Polko JK, van Zanten M, van Rooij JA, Marée AFM, LACJ Voesenek, Peeters AJM, Pierik R (2011) Ethylene-induced differential petiole growth in Arabidopsis thaliana involves local microtubule reorientation and cell expansion. New Phytol. doi: 10.1111/j.1469-8137.2011.03920.x

Ritsema T, van Zanten M, Leon-Reyes A, Voesenek LACJ, Millenaar FF, Pieterse CMJ, Peeters AJM (2010) Kinome profiling reveals an interaction between jasmonate, salicylate and light control of hyponastic petiole growth in Arabidopsis thaliana. PLoS One 5:e14255

Spoel SH, Koornneef A, Claessens SM, Korzelius JP, Van Pelt JA, Mueller MJ, Buchala AJ, Métraux JP, Brown R, Kazan K, Van Loon LC, Dong X, Pieterse CMJ (2003) NPR1 modulates crosstalk between salicylate- and jasmonate-dependent defense pathways through a novel function in the cytosol. Plant Cell 15:760-770

Thatcher LF, Anderson JP, Singh KB (2005) Plant defence responses: what have we learnt from Arabidopsis? Funct Plant Biol 32:1-19

van Zanten M, Millenaar FF, Cox MCH, Pierik R, Voesenek LACJ, Peeters AJM (2009a) Auxin perception and polar auxin transport are not always a prerequisite for differential growth. Plant Sig Behav 4:899-901

van Zanten M, Voesenek LACJ, Peeters AJM, Millenaar FF (2009b) Hormone- and light-mediated regulation of heat-induced differential petiole growth in Arabidopsis thaliana. Plant Physiol 151:1446-1458

van Zanten M, Pons TL, Janssen JAM, Voesenek LACJ, Peeters AJM (2010a) On the relevance and control of leaf angle. Crit Rev Plant Sci 29:300-316

van Zanten M, Snoek LB, Van Eck-Stouten E, Torii KU, Voesenek LACJ, Peeters AJM, Millenaar FF (2010b) Ethylene-induced 
hyponastic growth in Arabidopsis thaliana is controlled by ERECTA. Plant J 61:83-95

Vasseur F, Pantin F, Vile D (2011) Changes in light intensity reveal a major role for carbon balance in Arabidopsis responses to high temperature. Plant Cell Environ 34:1563-1576
Voesenek LACJ, Colmer TD, Pierik R, Millenaar FF, Peeters AJM (2006) How plants cope with complete submergence. New Phytol 170:213-226 